\title{
Inquisitive Semantics: Two Possibilities for Disjunction
}

\author{
Jeroen Groenendijk* \\ ILLC/Department of Philosophy \\ University of Amsterdam \\ j.a.g.groenendijk@uva.nl \\ http://home.medewerker.uva.nl/j.a.g.groenendijk
}

\begin{abstract}
We introduce an inquisitive semantics for a language of propositional logic, where the interpretation of disjunction is the source of inquisitiveness. Indicative conditionals and conditional questions are treated on a par both syntactically and semantically. The semantics comes with a new logical-pragmatical notion which judges and compares the compliance of responses to an initiative in inquisitive dialogue.
\end{abstract}

\section{Introduction}

In this paper we introduce an inquisitive semantics for a language of propositional logic. In inquisitive semantics, the semantic content of a sentence is not identified with its informative content. Sentences are interpreted in such a way that they can embody both data and issues.

The propositional language for which we define the semantics is a hybrid inquisitive language. By this we mean that there is no distinction in the syntax of the logical language between declarative and interrogative sentences, but questions and assertions can be characterized in semantic terms, next to hybrid sentences, which are both informative and inquisitive. Plain contingent disjunctions will count as such.

The language will enable us not only to express simple polar questions such as: "Will Bea go to the party?", but also conditional questions like: "If Alf goes to the party, will Bea go as well?", and alternative questions like: "Will Alf go to the party, or Bea?".

\footnotetext{
* I presented material related to this paper at several occasions in the past two years, including my Semantics and Pragmatics classes. I thank everyone involved in these events, especially my students, for helping me to get clearer about things. I owe special thanks to Floris Roelofsen for his many comments on many earlier drafts; to Frank Veltman, who insisted on improving the selling points; to Kata Balogh, who works with me on inquisitive pragmatic matters; and, last but not least, my close companion in this project, Salvador Mascarenhas, who discovered the inquisitive behavior of disjunction in an older version of the semantics. I am also grateful to the two anonymous referees for their helpful comments, and apologize for not having been able to respond more adequately to their remarks.
} 
The natural use of an inquisitive language lies in dialogues that have the purpose of raising and resolving issues. We will introduce a logical notion that judges whether a sentence $\varphi$ is compliant to a sentence $\psi$. We look upon $\varphi$ as a response to an initiative $\psi$, and require $\varphi$ to be strictly and obediently related to $\psi$. Compliance is a very demanding notion of dialogue coherence. ${ }^{1}$

\section{Two Possibilities for Disjunction}

The present paper explores an inquisitive logic for the simple artificial language of propositional logic. Hence, the logical notion of compliance that the semantics gives rise to applies to artificial inquisitive dialogues within this toy language. Nevertheless, such a logical semantical enterprise would be idle if we cannot make some connection with dialogue coherence relations in natural language.

I will discuss a small set of examples where I hope to illustrate that not only interrogatives, but also certain indicative sentences, in particular disjunctions, can be inquisitive. The empirical support for this comes from observations concerning compliant responses to such sentences. ${ }^{2}$

If an utterance is inquisitive and embodies an issue, a most compliant response to it is an utterrance that resolves the issue. If an indicative sentence allows for responses that are like answers to a question, are like typical responses to an interrogative sentence, I take this to indicate that the sentence has inquisitive semantic content.

To be able to contrast inquisitive with non-inquisitive sentences, with purely informative sentences, I also have to make certain assumptions about characteristic reponses to non-inquisitive sentences. I take it that if an utterance is purely informative, a response to it may typically be preceded by an interjection that reports the attitude of the responder to the information that was provided. We can take the function of that to establish the status of the information in relation to the common ground.

Consider the simple disjunction in (1), and the interrogative in (2).

(1) Alf or Bea will go to the party.

(2) Will Alf or Bea go to the party?

It is generally acknowledged that the interrogative in (2) has different intonation patterns. On one pattern, the two responses in (3) are the most compliant ones.

\footnotetext{
${ }^{1}$ In Groenendijk (1999), I defined a similar notion which I called 'licensing'. I switched to the term 'compliance', because it has a negative ring to it, and thus communicates more clearly that being non-compliant can easily be a virtue rather than a vice.

2 A difficulty I have to face is that in discussing examples I have to rely on intuitions about what counts as a compliant response to an initiative in a dialogue, just as much as in declarative semantics we have to rely on intuitions about entailment relations. The latter have been shaped and sharpened by the practices of the semanticist community. As for intuitions about compliance, I can only hope that you can share mine to at least some extent.
} 
(3) a. Yes. Alf or Bea will go to the party.

b. No. Neither of them will go.

Of course, though not answers in this case, these are equally good responses to the indicative sentence in (1), confirming and rejecting what (1) says, respectively, with opposite effects on the common ground status of the information provided by (1).

On another intonation pattern, perhaps the more common one, $(2)$ is not a yes/no-question, but has an alternative question interpretation, which has two different most compliant responses, one of which is (4).

(4) Bea will go.

The crucial thing to note is that the indicative disjunction in (1) also has an intonation pattern, on which (4) and its alternative are fully compliant responses. That is not to say that something that amounts to (4) could not be given as a reaction to (1), when it has the intonation pattern where the responses in (3) are the most expected ones, but the most 'appropriate' way to do it, would then be by means of (5) rather than (4).

(5) Yes. (In fact,) Bea will go.

First (1) as such is confirmed, establishing that the information it provides can enter the common ground, which sort of clears the way to elaborate on this by stating one of the disjuncts of (1), thus addressing a further issue, not embodied by (1) as such. Much the same holds for (5) in response to (2) on its yes/noreading.

Subtle as it may be, I take this to be evidence that like the interrogative in (2) is semantically ambiguous between a yes/no-reading and an alternative question reading, the indicative in (1) is semantically ambiguous between a purely informative reading, and a hybrid reading under which (1) is inquisitive as well. The ambiguity of the question in (2) can then be traced back to an underlying inquisitiveness ambiguity of disjunction as such.

The two readings, which can be set apart by intonation, have different effects on what counts as a compliant response, just as much as in declarative semantics different readings of a sentence can have different effects on entailment relations.

I will argue in the final section of the paper, that a hybrid disjunction and the corresponding alternative question also implicate that not both disjuncts hold, and that the logical notion of compliance can offer an explanation for that.

\section{$3 \quad$ Hybrid Propositional Syntax}

The syntax of our propositional language is stated in a reasonably standard way.

Definition 1 (Hybrid Propositional Syntax). Let $\wp$ be a finite set of propositional variables. ${ }^{3}$ The set of sentences of $L_{\wp}$ is the smallest set such that:

\footnotetext{
${ }^{3}$ The assumption that the set of atoms is finite only plays a marginal role in this paper,
} but is of importance, e.g., for proving functional completeness. See footnote 8. 
1. If $p \in \wp$, then $p \in L_{\wp}$

2. $\perp \in L_{\wp}$

3. If $\varphi \in L_{\wp}$ and $\psi \in L_{\wp}$, then $(\varphi \rightarrow \psi) \in L_{\wp}$

4. If $\varphi \in L_{\wp}$ and $\psi \in L_{\wp}$, then $(\varphi \wedge \psi) \in L_{\wp}$

5. If $\varphi \in L_{\wp}$ and $\psi \in L_{\wp}$, then $(\varphi \vee \psi) \in L_{\wp}$

The non-standard nature of the language, the fact that it is a hybrid inquisitive language, becomes apparent in the last two items in the following small list of notation conventions.

Definition 2 (Notation Conventions).

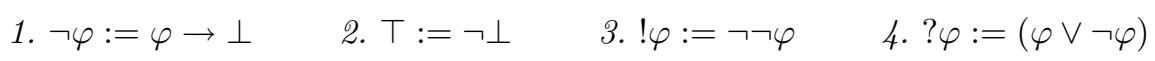

The interpretation of negation, which is standardly defined in terms of implication and the falsum, will be such that $\neg \varphi$ is never inquisitive, is always an assertion. Hence, whether $\varphi$ is inquisitive or not, $! \varphi$ will be non-inquisitive and will only be equivalent with $\varphi$, if $\varphi$ is an assertion.

The interpretation of disjunction will be such that although $\varphi \vee \neg \varphi$ is never informative, $\varphi \vee \neg \varphi$ is inquisitive as soon as $\varphi$ is contingent. This means that $\varphi \vee \neg \varphi$ and $T$ are not equivalent, since given the way in which $T$ is defined, it is not only non-informative but also non-inquisitive. Since $\varphi \vee \neg \varphi$ can only be inquisitive it makes sense to mark it as the question ? $\varphi$.

Another non-standard feature of the language is that unlike in classical propositional logic, conjunction, disjunction and implication are not interdefinable in the usual way with the aid of negation. ${ }^{4}$

There is no perfect match between our toy logical language and natural language. For example, the logical syntax allows for the negation of questions, questions occurring as the antecedent of conditional sentences, and disjunctions of questions. I will not discuss such disputable features of the logic in the present paper.

\section{Inquisitive Semantics}

We state the semantics for a language $L_{\wp}$ relative to a set $W_{\wp}$ of suitable possible worlds for $L_{\wp}$, where a world $w \in W_{\wp}$ is a valuation function with the set of propositional variables $\wp$ as its domain and the two values $\{1,0\}$ as its range. ${ }^{5}$

For a declarative language, a standard way to define the interpretation of the sentences of the language is by the notion $w \models \varphi$, which can be read as: $w$ confirms the information provided by $\varphi$. This will not suffice to interpret our hybrid inquisitive language, where sentences may not only provide information, but may also embody issues.

\footnotetext{
${ }^{4}$ In its non-standard features, inquisitive logic bears resemblances to intuitionistic logic. Inquisitive logic is a so-called intermediate logic. Except for one more footnote, I will not address the matter. See Mascarenhas (2008).

${ }^{5}$ We will often suppress the subscript $\wp$ on $L$ and $W$.
} 
The minimal way to deal with this is to evaluate sentences relative to pairs of worlds, and define the interpretation of the language in terms of the notion $(w, v) \models \varphi$, which we read as $w$ and $v$ agree upon $\varphi$. We take this to mean that both $w$ and $v$ confirm the information provided by $\varphi$, and that if $\varphi$ embodies an issue the answer to it can be the same in $w$ and $v$. In case of $(w, w) \models \varphi$ this boils down to $w$ confirms the information provided by $\varphi .^{6}$

Definition 3 (Inquisitive Semantics). Let $\varphi \in L_{\wp}$, and $w, v \in W_{\wp}$.

$$
\begin{aligned}
& \text { 1. }(w, v) \models p \text { iff } w(p)=1 \text { and } v(p)=1 \\
& \text { 2. }(w, v) \models \perp \\
& \text { 3. }(w, v) \models(\varphi \vee \psi) \text { iff }(w, v) \models \varphi \text { or }(w, v) \models \psi \\
& \text { 4. }(w, v) \models(\varphi \wedge \psi) \text { iff }(w, v) \models \varphi \text { and }(w, v) \models \psi \\
& \text { 5. }(w, v) \models(\varphi \rightarrow \psi) \text { iff for all pairs } \pi \in\{w, v\}^{2}: \text { if } \pi \models \varphi \text {, then } \pi \models \psi
\end{aligned}
$$

The definition has pretty familiar looks, ${ }^{7}$ except for the clause for implication, which quantifies over the four pairs $(w, v),(v, w),(v, v)$, and $(v, v)$. It can easily be read from the other clauses that to consider $(v, w)$ next to $(w, v)$ is redundant.

We will discuss implication more extensively later, but note that to inspect whether $(w, v)$ agree upon $\varphi \rightarrow \psi$, we not only check whether if $(w, v)$ agree on $\varphi,(w, v)$ agree upon $\psi$ as well. Also in case $(w, v)$ do not agree upon $\varphi$, because $w$ confirms the information provided by $\varphi$ whereas $v$ does not (or the other way around), we still keep on checking in that case, whether $w$ (or $v$ ) also confirms the information provided by $\psi$.

Although, as I announced in the introduction, we will see later that the semantics gives rise to a new logical notion of compliance that rules the use of the inquisitive language in dialogue, an orthodox notion of entailment in terms of agreement suggests itself as well.

\section{Definition 4 (Entailment).}

$$
\begin{aligned}
& \varphi_{1}, \ldots, \varphi_{n} \models \psi \text { iff } \\
& \quad \forall w, v \in W: \text { if }(w, v) \models \varphi_{1} \text { \& } \ldots \mathscr{B}(w, v) \models \varphi_{n}, \text { then }(w, v) \models \psi .
\end{aligned}
$$

The notion of entailment is well-behaved and has interesting properties. To note one, from the way in which implication is defined, it immediately follows that under the inquisitive interpretation of the language the following fact holds.

\footnotetext{
${ }^{6}$ The present format of the semantics was suggested to me by Balder ten Cate. The semantics can also be generalized by stating the interpretation relative to sets of possible worlds of arbitrary size. The resulting semantics is richer, and the logic is closer to intuitionistic logic.

7 The clause for disjunction may also look familiar to you if (like Robert van Rooij, thanks) you have read David Lewis' paper: 'Whether' report (Lewis (1982)). There, Lewis considers to treat whether $A$ or $B$ clauses as wheth $A$ or wheth $B$ along the following lines: $\models_{i, j}$ whether $A$ or $B$ iff $\models_{i, j}$ wheth $A$ or $\models_{i, j}$ wheth $B$, where $\models_{i, j}$ wheth $A$ iff $\models_{i} A$ and $\models_{j} A$. The notion $\models_{i, j}$ is conceived of as an application of the technique of double indexing.
} 
Fact 1. $\varphi \models \psi$ iff $\models \varphi \rightarrow \psi$

Logical equivalence of two formulas is defined as usual as mutual entailment.

Definition 5 (Equivalence). $\varphi \Leftrightarrow \psi$ iff $\varphi=\psi \& \mathcal{E} \psi=\varphi$

With these logical notions in place, we turn to the discussion of the semantics.

\subsection{Informativeness and Inquisitiveness}

The first clause in Def. 3 implies that a propositional variable $p$ does not embody an issue. The definition says that to see whether $w$ and $v$ agree upon $p$, it is sufficient to see whether $w(p)=v(p)=1$. We have that $(w, v) \models p$ iff $(w, w) \models p$ and $(v, v) \models p$.

This is different for a disjunction like $p \vee q$. There we can have that $(w, w) \models$ $p \vee q$ and $(v, v) \models p \vee q$, whereas $(w, v) \not \models p \vee q$, as can be shown as follows.

Let $w$ be a world where $w(p)=1 \& w(q)=0$, and $v$ a world where $v(p)=0 \&$ $v(q)=1$. Then we have that $(w, w) \models p$ and $(v, v) \models q$, and hence, according to clause 3 of Def. 3, both $(w, w) \models p \vee q$ and $(v, v) \models p \vee q$.

At the same time we have that $(w, v) \not \models p$, because $v(p)=0$; and $(w, v) \not \models q$, since $w(q)=0$. According to the definition, this means that $(w, v) \not \models p \vee q$. So, we have shown that, unlike in the case of atomic sentences, there are worlds $w$ and $v$ such that $(w, w) \mid=p \vee q$ and $(v, v) \models p \vee q$, whereas $(w, v) \not \vee p \vee q$.

Two such worlds $w$ and $v$ do not agree upon $p \vee q$, because although $w$ and $v$ both confirm the information provided by $p \vee q$, the answer to the issue that $p \vee q$ embodies, the issue whether $p$ or $q$, is different in $w$ and $v$, in $w$ the answer is $p$, in $v$ it is $q$.

We have just shown that an atomic sentence $p$ is not inquisitive, and that a disjunction like $p \vee q$ is inquisitive, according to the following definition:

Definition 6 (Consistency, Informativeness, and Inquisitiveness).

1. $\varphi$ is consistent iff $\exists w \in W:(w, w) \models \varphi$.

2. $\varphi$ is informative iff $\exists w \in W:(w, w) \not=\varphi$.

3. $\varphi$ is inquisitive iff $\exists w, v \in W:(w, w) \models \varphi \quad \mathcal{G}(v, v) \models \varphi \& \mathcal{G}(w, v) \not \models \varphi$.

We also define:

(a) $\varphi$ is hybrid iff $\varphi$ is informative and $\varphi$ is inquisitive.

(b) $\varphi$ is contingent iff $\varphi$ is consistent, and $\varphi$ is inquisitive or informative.

What inquisitiveness of $\varphi$ requires is that there are pairs of worlds that satisfy the information provided by $\varphi$ (which implies that $\varphi$ is consistent), but where the two worlds differ in their answer to an issue embodied by $\varphi$, which implies that $\varphi$ indeed does embody an issue, otherwise two such worlds could not be found.

As we have seen, informativeness and inquisitiveness do not exclude each other, $p \vee q$ is both informative and inquisitive, and hence semantically hybrid.

Given the way the interpretation of $\perp$ is defined, $\perp$ is not contingent, since it is inconsistent. And $T$ is not contingent either, because $\perp$ is neither inquisitive nor informative. 


\subsection{Negations and Assertions}

Before we turn to negation, we note that on the basis of the informal description of $(w, v) \models \varphi$ in terms of agreement, we may expect the following to hold, which indeed it does, given the way the semantics is defined:

Fact 2 (Symmetry and Reflexive Closure of Agreement).

1. $\forall w, v \in W:(w, v) \models \varphi \Rightarrow(v, w) \models \varphi$.

2. $\forall w, v \in W:(w, v) \models \varphi \Rightarrow(w, w) \models \varphi \& \mathcal{G}(v, v) \models \varphi$.

The proof runs by induction on the complexity of $\varphi .^{8}$

From the definition of inquisitiveness and the last item in Fact 2, the following follows immediately.

\section{Fact 3 (Non-Inquisitiveness).}

$$
\varphi \text { is not inquisitive iff } \forall w, v \in W:(w, v) \models \varphi \Leftrightarrow(w, w) \models \varphi \mathscr{G}(v, v) \models \varphi \text {. }
$$

Let us now consider $\neg \varphi$ which abbreviates $\varphi \rightarrow \perp$. Clause 5 of Def. 3 tells us that for $w$ and $v$ to agree upon $\varphi \rightarrow \perp$, it should hold for the four pairs $(w, v)$, $(v, w),(w, w)$, and $(v, v)$, that if such a pair agrees on $\varphi$ it agrees on $\perp$. The interpretation of $\perp$ tells us that no pair agrees on $\perp$. Hence, For $w$ and $v$ to agree upon $\varphi \rightarrow \perp$, it should hold that $(w, v) \not \models \varphi,(v, w) \not \models \varphi,(w, w) \not \models \varphi$, and $(v, v) \not \varphi$. Given Fact 2 and the way negation is introduced in the language, this boils down to:

Fact 4 ( $\neg$ Negation). $(w, v) \models \neg \varphi$ iff $(w, w) \not \models \varphi \&(v, v) \not \models \varphi$.

Two worlds agree upon a negation as soon as neither of the two confirms the information provided by $\varphi$. As we saw to be the case for atomic sentences, negations embody no issue. From Facts 1 and 2 it immediately follows that:

Fact 5 (Negation). $\neg \varphi$ is not inquisitive.

That negations are never inquisitive, is of course behind the fact that disjunction, conjunction and implication are not interdefinable in the usual way with the aid of negation. Disjunction is the indispensable source of inquisitiveness in the language. And if we were to define implication and conjunction in terms of disjunction and negation we do not in general obtain the interpretation now assigned by the semantics to formulas of these forms.

Since $! \varphi$ is defined as double negation, $! \varphi$ is not inquisitive. And we can write:

\footnotetext{
${ }^{8}$ In inquisitive update semantics, a state $\sigma$ for $L_{\wp}$ is defined as a reflexive and symmetric relation on a subset of $W_{\wp}$. In the semantics we recursively define the effect of updating $\sigma$ with $\varphi, \sigma[\varphi]$. The relation with the present semantics is given by: $\sigma[\varphi]=\{(w, v) \in \sigma \mid(w, v) \models \varphi\}$. Salvador Mascarenhas proved a Functional Completeteness Theorem, which says that for any two states $\sigma$ and $\sigma^{\prime}, \sigma^{\prime} \subseteq \sigma \subseteq W_{\wp}^{2}$ : there is a finite sequence of sentences $\varphi_{1}, \ldots, \varphi_{n} \in L_{\wp}$ such that $\sigma\left[\varphi_{1}\right] \ldots\left[\varphi_{n}\right]=\sigma^{\prime}$. We don't need the full language to achieve this, $\{\neg, \vee\}$ suffices. Since conjunction corresponds to sequencing, if we add $\wedge$, we can move from any state to any of its substates with a single formula of the language. The assumption we made that $\wp$ is finite, is essential for the functional completeness proof. (See Mascarenhas (2008).)
} 
Fact 6 (!Assertion). $(w, v) \models ! \varphi$ iff $(w, w) \models \varphi \&(v, v) \models \varphi$.

This means that for any formula $\varphi, ! \varphi$ delivers its interpretation in classical logic. From Facts 3 and 6 it follows that every non-inquisitive sentence can be written as an assertion:

Fact 7 (Assertion). ! $\varphi \Leftrightarrow \varphi$ iff $\varphi$ is not inquisitive.

Given this fact, we will often refer to non-inquisitive sentences as assertions.

Note that Fact 7 tells us that the law of triple negation holds. Since negation is not inquisitive, ! $\neg \varphi \Leftrightarrow \neg \varphi$. And iteration of ! is superfluous: !! $\varphi \Leftrightarrow ! \varphi$.

Also, since ! $(p \vee q)$ is not inquisitive, it is not equivalent with the hybrid disjunction $p \vee q$. The assertion ! $(p \vee q)$ embodies no issue, ! $(p \vee q)$ just embodies the truthconditional content of disjunction in classical logic.

Remember the discussion in Section 2, where we observed that the English indicative disjunction (1), like its interrogative sister (2), is prosodically ambiguous between a yes/no-interpretation, and an alternative interpretation. As for the latter, for both (1) and (2), the hybrid disjunction $p \vee q$ suggests itself as a proper translation. As for the yes/no-interpretation of the indicative (1), the assertion! $(p \vee q)$ seems to cover its meaning. And if we take the disjunction $!(p \vee q) \vee \neg(p \vee q)$, i.e., ?! $(p \vee q)$ we get a polar question that suits (2) on its yes/no-reading. This brings us to questions.

\subsection{Questions}

Consider the atomic question ? $p$, which abbreviates $p \vee \neg p$. The interpretation of disjunction tells us that $(w, v) \models p \vee \neg p$ iff $(w, v) \models p$ or $(w, v) \models \neg p$, both worlds agree upon $p$ or both worlds agree upon $\neg p$. This means that $(w, v) \models ? p$ iff $w(p)=v(p)=1$ or $w(p)=v(p)=0$. From this it is clear that ? $p$ is not informative, and is inquisitive, $p \vee \neg p$ is contingent, it is an inquisitive question.

Given the interpretation of disjunction and the interpretation of negation given in Fact 4, we can write:

Fact 8 (?Questions). $(w, v) \models ? \varphi$ iff $(w, v) \models \varphi$ or $(w, w) \not \models \varphi \&(v, v) \not \models \varphi$.

If we consider $(w, w) \models ? \varphi$, we get that $(w, w) \models ? \varphi$ iff $(w, w) \models \varphi$ or $(w, w) \not \models$ $\varphi$, which trivially holds, hence $\forall w:(w, w) \models ? \varphi$. Given how informativeness is defined, the first item in the next fact holds, from which the second item immediately follows.

Fact 9 (Questions). ? $\varphi$ is not informative $\mathbb{G} ? \varphi \Leftrightarrow \varphi$ iff $\varphi$ is not informative.

Given this fact, we will often refer to non-informative sentences as questions. (Note that since $T$ is neither informative nor inquisitive, it counts both as a (non-informative) assertion, and as a (non-inquisitive) question.)

The last item in Fact 9 implies that iteration of ? is superfluous: ?? $\varphi \Leftrightarrow ? \varphi$. The fact that iteration of both! and ? are superfluous, makes it easy to state things in general about assertions and questions. 


\subsection{Conditionals: Divide and Conquer}

The two specific cases of conditionals with a question, and conditionals with an assertion as consequent, behave more standardly than the clause for implication in Def. 3 might suggest. ${ }^{9}$

Fact 10 (Conditional Questions and Conditional Assertions).

1. $(w, v) \models \varphi \rightarrow ? \psi$ iff $(w, v) \models \varphi \Rightarrow(w, v) \models ? \psi$

2. $(w, v) \models \varphi \rightarrow ! \psi$ iff $(w, w) \models \varphi \rightarrow \psi$ and $(v, v) \models \varphi \rightarrow \psi$

Clause 5 in Def. 3 requires for $(w, v) \models \varphi \rightarrow \psi$ that it holds for each of the pairs $\pi$ we can form from $w$ and $v$, i.e., $(w, v),(v, w),(w, w)$, and $(v, v)$ that: if $\pi \models \varphi$, then $\pi \models \psi$. Given symmetry of agreement (Fact 2), among those four, we can dismiss $(v, w)$.

Furthermore, since ? $\psi$ is not informative, i.e., $\forall w:(w, w) \models ? \psi$, in evaluating $\varphi \rightarrow ? \psi$, we can dismiss the two identity pairs as well. This also means that if $\psi$ is not informative, then neither is $\varphi \rightarrow \psi$.

Conversely, since ! $\psi$ is not inquisitive, i.e., $(w, v) \models \psi$ iff $(w, w) \models \psi$ \& $(v, v) \models \psi$ (Fact 3 ), in evaluating $\varphi \rightarrow ! \psi$, we only have to consider the two identity pairs. This also means that if $\psi$ is not inquisitive, $\varphi \rightarrow \psi$ is not inquisitive either, and behaves like classical material implication:

Fact 11 (Non-Inquisitive Conditionals). $\varphi \rightarrow ! \psi \Leftrightarrow !(\varphi \rightarrow \psi)$

So, we have seen how the two specific cases of conditionals with non-inquisitive and non-informative consequents behave more standardly than the clause for implication in Def. 3 might suggest. But we can actually show that any conditional reduces to a combination of these two simple cases.

The following fact tells us that every sentence $\varphi$ can be divided in a theme $? \varphi$ and a rheme $! \varphi$.

Fact 12 (Division). $\varphi \Leftrightarrow ? \varphi \wedge ! \varphi$

From this it immediately follows that every conditional $\varphi \rightarrow \psi$ can be written as $\varphi \rightarrow(? \psi \wedge ! \psi)$. Next, we use the following distribution fact.

Fact 13 (Distribution 1). $\varphi \rightarrow(\psi \wedge \chi) \Leftrightarrow(\varphi \rightarrow \psi) \wedge(\varphi \rightarrow \chi)$

This allows us to rewrite the conditional $\varphi \rightarrow(? \psi \wedge ! \psi)$ as the conjunction of conditionals $(\varphi \rightarrow ? \psi) \wedge(\varphi \rightarrow ! \psi)$. Finally, applying the equivalence in Fact 11 to the second conjunct, we arrive at the following fact.

Fact 14 (Conditional Division). $\varphi \rightarrow \psi \Leftrightarrow(\varphi \rightarrow ? \psi) \wedge !(\varphi \rightarrow \psi)$

Any conditional can be rewritten as the conjunction of a conditional question and a classical material implication. ${ }^{10}$

\footnotetext{
9 The semantics of conditional questions presented here is similar to the one proposed in Velissaratou (2000). What is new is that in our hybrid inquisitive language we have a single uniform interpretation of implication that deals both with conditional questions and conditional assertions.

10 Actually, we may take this to mean that the theme of a plain conditional, the question on the background, is the corresponding conditional question (rather than the
} 


\subsection{Disjunctive Antecedents}

Consider the simplest example $p \rightarrow ? q$ of a conditional question. We get that $(w, v) \models p \rightarrow ? q$ iff $w(p)=v(p)=1 \Rightarrow w(q)=v(q)$. I.e., either $w(p)=v(p)=$ $1 \Rightarrow w(q)=v(q)=1$ or $v(p)=w(p)=1 \Rightarrow w(q)=v(q)=0$. Which means that $p \rightarrow ? q$ is equivalent with the disjunction $(p \rightarrow q) \vee(p \rightarrow \neg q)$. This is a special instance of the following equivalence: ${ }^{11}$

Fact 15 (Mascarenhas Equivalence). $! \varphi \rightarrow(\psi \vee \chi) \Leftrightarrow(! \varphi \rightarrow \psi) \vee(! \varphi \rightarrow \chi)$

This equivalence does not hold generally also in case of inquisitive antecedents. The following pair of examples is a case in point: $(p \vee q) \rightarrow ? r \Leftrightarrow !(p \vee q) \rightarrow ? r$. With the Mascarenhas Equivalence, $!(p \vee q) \rightarrow ? r$ corresponds to a disjunction of two assertions: $((p \vee q) \rightarrow r) \vee((p \vee q) \rightarrow \neg r)$. We will show that $(p \vee q) \rightarrow ? r$ corresponds to a longer disjunction of four assertions.

First we note another distribution fact.

Fact 16 (Distribution 2). $(\varphi \vee \psi) \rightarrow \chi \Leftrightarrow(\varphi \rightarrow \chi) \wedge(\psi \rightarrow \chi)$

This means that $(p \vee q) \rightarrow ? r$ is equivalent to $(p \rightarrow ? r) \wedge(q \rightarrow ? r)$, which is Mascarenhas-equivalent to $((p \rightarrow r) \vee(p \rightarrow \neg r)) \wedge((q \rightarrow r) \vee(q \rightarrow \neg r))$, to which we apply the (last) distribution fact:

\section{Fact 17 (Distribution 3).}

$$
(\varphi \vee \psi) \wedge(\chi \vee \theta) \Leftrightarrow(\varphi \wedge \chi) \vee(\varphi \wedge \theta) \vee(\psi \wedge \chi) \vee(\psi \wedge \theta)
$$

This gives us four disjuncts, two of which are $(p \rightarrow r) \wedge(q \rightarrow r)$ and $(p \rightarrow$ $\neg r) \wedge(q \rightarrow \neg r)$, which by Fact 16 reduce to the first two of the following four disjuncts, which together are equivalent with $(p \vee q) \rightarrow ? r$ :

$((p \vee q) \rightarrow r) \vee((p \vee q) \rightarrow \neg r) \vee((p \rightarrow r) \wedge(q \rightarrow \neg r)) \vee((p \rightarrow \neg r) \wedge(q \rightarrow r))$

What we have arrived at, is that there are, as we will call them, four possibilities for the sentence $(p \vee q) \rightarrow ? r$. In this case, since the sentence is an inquisitive question, the four possibilities correspond to four possible answers. ${ }^{12}$

corresponding questioned conditional). Thus, inquisitive semantics may be taken to give a logical explanation for the idea ventured in the first sentence of Ramsey's famous footnote, known as the Ramsey Test: (Ramsey (1931))

If two people are arguing "If $p$ will $q$ ?" and are both in doubt as to $p$, they are adding $p$ hypothetically to their stock of knowledge and arguing on that basis about $q$; so that in a sense "If $p, q$ " and "If $p, \neg q$ " are contradictories.

These two 'contradictories' are the two answers to the conditional question that we just found to be the theme of a conditional. See also Grice's paper on 'Indicative Conditionals' in Grice (1989).

11 The hard part of this equivalence, from left to right (and with $\neg \varphi$ instead of ! $\varphi$ ), is known as the Kreisel-Putnam Axiom and corresponds to an admissible rule in intuitionistic propositional logic. Salvador Mascarenhas has proved that it is also valid in inquisitive propositional logic. This result is crucial in obtaining a disjunctive normal form. (See footnote 14.)

${ }^{12}$ We discovered this nice feature of the semantics by surprise. Tikitu de Jager programmed the semantics. The program spits out the possibilities (see below) that 


\subsection{Possibilities}

Given the properties of $(w, v) \models \varphi$, as stated in Fact 2, the relation between worlds of 'to agree upon a sentence $\varphi$ ', corresponds to a set of sets of worlds. ${ }^{13}$

Definition 7 (Possibilities). Let $\varphi \in L_{\wp} . P$ is a possibility for $\varphi$ in $W_{\wp}$ iff

1. $P \subseteq W_{\wp} \& \forall \forall w, v \in P:(w, v) \models \varphi$; and

2. $\forall P^{\prime} \subseteq W_{\wp}$ : if $P \subseteq P^{\prime} \quad \& \forall \forall, v \in P^{\prime}:(w, v)=\varphi$, then $P^{\prime}=P$.

A possibility for a sentence $\varphi$ is a largest set $P$ of worlds (a proposition), such that for any two worlds $w, v \in P: w$ and $v$ agree upon $\varphi .^{14}$

A sentence $\varphi$ is inquisitive iff there is more than one possibility for $\varphi ; \varphi$ is not inquisitive iff there is a single possibility for $\varphi$. The set of possibilities for $\perp$ in $W$ is $\{\emptyset\}$. The set of possibilities for $\top$ is $\{W\}$. A sentence $\varphi$ is informative iff the union of the set of possibilities for $\varphi$ does not equal $W$.

If there is more than one possibility for a sentence $\varphi$, then each possibility corresponds to a proposition that fully resolves the issue embodied by $\varphi$. Unions of (some but not all) possibilities for $\varphi$ correspond to propositions that partially resolve the issue embodied by $\varphi$.

\section{$5 \quad$ Inquisitive Logic}

If we ask ourselves what the natural purpose of an inquisitive language is, the obvious answer is: to raise and resolve issues; a purpose best suited in dialogue. Then a natural task for a logic that comes with inquisitive semantics is to address moves in a dialogue concerned with cooperatively raising and resolving issues.

Following the lead of the 'normative' status of the logical notion of entailment in judging validity of argumentation, we can take inquisitive logical notions to judge 'correctness', or 'coherence', or 'compliance' of a response to an initiative in a cooperative inquisitive dialogue.

Here we can draw from general insights in dialogue studies. ${ }^{15}$ Two fundamental dialogue coherence relations for a response to an initiative are the following:

a formula gives rise to. This is particularly helpful for formulas with more than two propositional variables, which are hard to picture. We ran the program on $(p \vee q) \rightarrow ? r$, expecting to get out the two possibilities for $!(p \vee q) \rightarrow ? r$. Panic struck when the program predicted four possibilities. But after analyzing what came out, the program - and the semantics - turned out to be right. There is a reading of the question: "If Alf or Bea goes to the party, will Chris go as well?", that has the four possible answers that Tikitu's program came up with.

${ }^{13}$ In terms of the notion of the possibilities for a sentence, we can also more accurately describe $(w, v) \models \varphi$ as $w$ and $v$ agree upon $\varphi$ iff there is a possibility for $\varphi$ to which both $w$ and $v$ belong.

14 Salvador Mascarenhas has proved that the set of possibilities for a sentence $\varphi$ can be syntactically characterized as a disjunction of assertions, where each assertion characterizes a possibility. Any sentence can be transformed into its Inquisitive Disjunctive Normal Form, which has this property. (Mascarenhas (2008))

15 See, e.g., Asher \& Lascarides (1998), Hulstijn (2000), and Roberts (1996). 


\section{Two Dialogue Coherence Relations}

(i) Answer an issue raised by an initiative (informative relation); or

(ii) Replace the issue by an easier to answer subissue (inquisitive relation).

The inquisitive option is second choice, a cooperative responder takes recourse to it only if he lacks the information for even a partial fulfilment of the first option. And note that if the initiative is a question, we may assume that the initiator certainly has no full answer to it, but she just may have a bit of a partial answer to her own question. Hence, it can make sense for the responder to ask a counter question, if only because when that bit of the issue were resolved, it may become possible for him to provide a full(er) answer to the initial question. ${ }^{16}$

Of course, the less inquisitive such a counter question is, the better the chances are that this bit of the original bigger issue, turns out not to be an issue for the initiator.

If we go from here, then one can take it to be the case, that the general direction an inquisitive dialogue strives for, is to move from less informed to more informed situations, and from more inquisitive to less inquisitive situations.

If we look at entailment from this perspective, we see that more informativeness of $\varphi$ as compared to $\psi$ is measured by $\varphi=\psi$, whereas less inquisitiveness of $\varphi$ as compared to $\psi$ runs in the opposite direction, and is measured by $\psi \models \varphi$.

It is not too difficult to design a logical relation that measures informativeness and inquisitiveness in these opposite direction in one go. We call it homogeneity.

Definition 8 (Homogeneity). $\varphi$ is at least as homogeneous as $\psi, \varphi \succeq \psi$ iff

1. For all $w \in W$ : if $(w, w) \models \varphi$, then $(w, w) \models \psi$, and

2. For all $w, v \in W$ : if $(w, w) \models \varphi \mathscr{G}(v, v) \models \varphi \mathscr{G}(w, v) \not \models \varphi$, then $(w, v) \not \models \psi$.

The second clause holds trivially for assertions, since the antecedent can never be the case. The first clause holds trivially for questions. The most essential features of homogeneity, and its hybrid relation to entailment, are listed below:

\section{Fact 18 (Homogeneity).}

1. If $\varphi \succeq \psi$, then $! \varphi \models ! \psi$

2. $! \varphi \succeq ! \psi$ iff ! $\varphi=! \psi$

3. If ! $\varphi \Leftrightarrow ! \psi$, then $\varphi \succeq \psi$ iff ? $\psi \models ? \varphi$

4. ? $\varphi \succeq ? \psi$ iff $? \psi \models ? \varphi$

5. $! \varphi \succeq ? \psi$

6. $\perp \succeq \varphi$

7. $\top \succeq ? \varphi$

${ }^{16}$ For similar reasons, it may also be sensible not to respond with a subissue, but with an objectively speaking unrelated question, which subjectively, for the responder, is related to the issue posed by the initiator. 
Although homogeneity gives the general direction an inquisitive dialogue strives for, as is particularly clear from the fact that any assertion is at least as homogeneous as any question (item 5 in the list), we need some more specific directions that tell us, e.g., which assertions are proper responses to which questions. The logical notion of relatedness, defined in terms of possibilities, does that.

Definition 9 (Relatedness). $\varphi$ is related to $\psi, \varphi \propto \psi$ iff

every possibility for $\varphi$ is the union of a subset of the set of possibilities for $\psi$.

Relatedness is defined generally for all kinds of sentences, but if $\varphi$ is an assertion, for which there is only a single possibility $P$, relatedness of $\varphi$ to $\psi$ requires that $P$ is the union of a subset of the set of possibilities for $\psi$, which, in case $\psi$ is inquisitive, is as close as you can logically expect to get, in characterizing partially resolving the issue raised by an initiative $\psi$.

By homogeneity we can measure whether the information contained in one sentence more fully resolves an issue, than the information contained in another sentence. Were it not for the borderline case of non-contingent $\perp$, which is more homogeneous than any contingent sentence, and is also related to every sentence, we could equate the most homogeneous related responses to an inquisitive initiative $\psi$ with those sentences $\varphi$ that completely resolve the issue $\psi$ embodies.

In other words, under the general constraint of contingency of a response, relatedness, combined with homogeneity, tells us how well a sentence does in resolving an issue. This concerns the informative dialogue coherence relation.

Concerning the inquisitive dialogue coherence relation, we get a similar story. First of all, if a question ? $\varphi$ is related to and at least as homogeneous as a question ? $\psi$, it is indeed guaranteed that ? $\varphi$ is at least as easy to answer as ? $\psi$.

Secondly, by homogeneity we can measure whether one question is a more minimal subissue of some issue, than another question is. Were it not for the borderline case of non-contingent $T$, which is the most homogeneous 'question', and is also related to every question, we could equate the most homogeneous related questions to some question $\psi$ ? with the minimal subquestions of $\psi$ ?

In other words, under the general constraint of contingency of a response, relatedness, combined with homogeneity, tells us how well a question does in replacing an issue by an easier to answer subissue. This concerns the inquisitive dialogue coherence relation.

We put our findings together in the following definition of compliance that deals with both dialogue coherence relations.

Definition 10 (Compliance). $\varphi$ is a compliant response to $\psi$ iff

1. $\varphi$ is contingent; and

2. $\varphi$ is related to $\psi$; and

3. $\varphi$ is at least as homogeneous as $\psi$. 
This qualitative notion of compliance embodies a comparative quantitative notion as well: among contingent sentences which are related to an initiative, homogeneity prefers more informative sentences, and among two equally informative sentences, it prefers less inquisitive sentences.

These are the borderline cases:

Fact 19 (Ultimate Compliance). Let $\psi$ be a contingent initiative.

1. $\varphi$ is a least compliant response to $\psi$ iff $\varphi$ is equivalent to $\psi$.

2. $\varphi$ is a most compliant response to $\psi$ iff there is a single possibilty $P$ for $\varphi$, such that $P$ is a possibility for $\psi$ as well.

3. If $\psi$ is a question, $\varphi$ is a most compliant non-informative response to $\psi$ iff $\varphi$ is a polar subquestion of $\psi$.

Note that in case the initiative is a polar question, the most compliant noninformative responses coincide with the least compliant responses. If the responder has no answer to a polar question, there is no significant move to make.

Similarly, in case the initiative is a contingent assertion, the least and most compliant responses coincide: repeating the initiative, at most rephrasing it a bit, is the only compliant move to make.

This is why we characterized compliance informally as strict and obedient relatedness. Compliance as such does not allow for critical responses. Logically speaking, it is just a small step to allow for critical responses: also permit compliance to the theme ? $\psi$ of an initiative $\psi$. Emotionally, though, say for a parent with a maturing child, this may be a big step. But that's another story.

\section{Conclusion: Inquisitive Pragmatics}

It will not have escaped your attention that the way the logical notion of compliance is defined bears resemblances to the Gricean Cooperation Principle and its Maxims of Quality, Relation and Quantity. This may give rise to the expectation that implicatures are around the corner.

Consider the example of a hybrid disjunction $p \vee q$ as an initiative. There are, up to equivalence, only two most compliant responses: $p$ and $q$. In particular, the more homogeneous sentence $p \wedge q$ is blocked, because it is not related to $p \vee q$. Apparently, according to the initiator, it does not count.

How can that be? We have taken it to be the case that a cooperative dialogue strives for more homogeneous situations. In principle, the initiator should be interested in obtaining the information whether $p \wedge q$ on top of the information that $p$ (or $q$ ). By blocking $p \wedge q$ as a response, the initiator suggests that: not both $p$ and $q$. And by responding with just $p$ to $p \vee q$, the responder signals that he goes along with that suggestion. Hence, his answer $p$ implicates that $\neg q$. Cooperatively, initiator and responder have agreed upon exclusive disjunction.

Of course, the responder may have reasons for not following the exclusiveness suggestion made by the initiator, just as he may have reasons not to accept the informative content, which excludes that neither $p$ nor $q$. In both cases, the 
responder opts for not being compliant. In such situations, the appropriate way to do this, is not to bluntly reject the information provided, with: "Neither $p$ nor $q$ !"; or to protest against the suggestion being made with: "Both $p$ and $q$ !". Compliant non-compliant responses are rather: "Well, actually, neither $p$ nor $q$ "; and: "Well, in fact, both $p$ and $q . "$, thus explicitly signalling awareness of the non-compliance of one's response. (See also the examples in Section 2.)

\section{$7 \quad$ References}

Aloni, M., A. Butler, and P. Dekker (eds): (2007), Questions in Dynamic Semantics, Elsevier, Oxford.

Asher, N, and A. Lascarides: (1998), 'Questions in Dialogue', Linguistics and Philosophy 21, pp. 237-309.

Grice, H.P.: (1989), Studies in the Ways of Words, Harvard University Press, Cambridge, Mass.

Groenendijk, J.: (1999), 'The Logic of Interrogation', in T. Matthews and D.L. Strolovitch (eds), The Proceedings of the Ninth Conference on Semantics and Linguistic Theory, CLC Publications, Ithaca, NY, pp. 109-126. Also in: Aloni, Butler, and Dekker (2007).

Hulstijn J.: (2000), Dialogue Models for Inquiry and Transaction, Ph.D. thesis, University of Twente.

Lewis, D.: (1982), ' 'Whether' Report', in his: Papers in Philosophical logic, Cambridge University Press, Cambridge, 1998, pp. 45-56.

Mascarenhas, S.: (2008), Inquisitive Logic, MSc in Logic thesis, ILLC, University of Amsterdam.

Ramsey, F. P. : (1931), 'General Propositions and Causality', in his: The Foundations of Mathematics and other Logical Essays, edited by R. B. Braithwaite, London: Kegan Paul, Trench \& Trubner, pp. 237-255.

Roberts, C.: (1996), 'Information Structure in Discourse', In J.H. Yoon and A. Kathol (eds), Working Papers in Linguistics 49, The Ohio State University, pp. 91-136.

Velissaratou, S.: (2000), Conditional Questions and Which-Interrogatives, MSc in Logic thesis, ILLC, University of Amsterdam. 